\title{
Mapping Labels in the Human Developing Visual System and the Evolution of Binocular Vision
}

\author{
Marie-Alexandra Lambot, ${ }^{1 *}$ Fanny Depasse, ${ }^{1 *}$ Jean-Christophe Noel, ${ }^{2}$ and Pierre Vanderhaeghen ${ }^{1}$ \\ ${ }^{1}$ Institute of Interdisciplinary Research (IRIBHM) and ${ }^{2}$ Department of Pathology, University of Brussels, Campus Erasme, B-1070 Brussels, Belgium
}

\begin{abstract}
Topographic representation of visual fields from the retina to the brain is a central feature of vision. The development of retinotopic maps has been studied extensively in model organisms and is thought to be controlled in part by molecular labels, including ephrin/Eph axon guidance molecules, displayed in complementary gradients across the retina and its targeting areas. The visual system in these organisms is primarily monocular, with each retina mapping topographically to its contralateral target. In contrast, mechanisms of retinal mapping in binocular species such as primates, characterized by the congruent, aligned mapping of both retinas onto the same brain target, remain completely unknown. Here, we show that the distribution of ephrin/Eph genes in the human developing visual system is fundamentally different from what is known in model organisms. In the human embryonic retina, EphA receptors are displayed along two gradients, sloping down from the center of the retina to its periphery. The EphB1 receptor, which controls the ipsilateral routing of retinal axons in the mouse, is expressed throughout the human temporal retina in coordination with the changes in EphA gene expression. In the dorsal lateral geniculate nucleus, ephrin- $\mathrm{A} / \mathrm{EphAs}$ are displayed along complementary retinotopic gradients. Our data point to an evolutionary model in which the coordinated divergence of the distribution of the receptors controlling retinal guidance and retinal mapping enabled the emergence of a fully binocular system. They also indicate that ephrin/Eph signaling plays a potentially major role in the development of neuronal connectivity in humans.
\end{abstract}

Key words: binocular vision; visual mapping; ephrin; retina; lateral geniculate nucleus; human

\section{Introduction}

Sperry (1963) was the first to propose that the generation of topographic maps was controlled by molecular labels displayed in complementary gradients across projecting and targeting areas. Since then, numerous studies centered on the mapping of retinal projections onto the tectum have validated this hypothesis. In particular, ephrin/Eph axon guidance factors were shown to act as genuine mapping labels to control the topography of retinal projections to the chick tectum or superior colliculus (SC) in the mouse (for review, see Flanagan and Vanderhaeghen, 1998; McLaughlin et al., 2003; Drescher, 2004). In this system, EphA receptors are displayed in a high temporal to low nasal gradient in the retina, whereas ephrin-As are displayed in a complementary, low anterior to high posterior, gradient in the tectum and SC. In addition ephrin-As in the retina and EphAs in the developing

Received March 1, 2005; revised June 2, 2005; accepted June 16, 2005.

This work was funded by grants from the Belgian National Fund for Scientific Research; the Belgian Fund for Medical Scientific Research; the Queen Elizabeth Medical Foundation; the Interuniversity Attraction Poles Programme, Belgian State, Federal Office for Scientific, Technical, and Cultural Affairs (P.V.); and the Fondation Erasme (M.-A.L.).P.V. and M.-A.L. are Research Associate and Research Fellow of the FNRS, respectively. We declare we have no competing financial interests. We thank $G$. Vassart for continuous support and interest and members of the Institute of Interdisciplinary Research for helpful discussions and advice. Help and support from the Departments of Obstetrics/Gynecology and Pathology of Erasme Hospital and the Institute of Pathology and Genetics of Loverval are deeply acknowledged.

${ }^{*}$ M.-A.L. and F.D. contributed equally to this work.

Correspondence should be addressed to Dr. Pierre Vanderhaeghen, Institute of Interdisciplinary Research, University of Brussels, Campus Erasme CP 602, Building C, Room 6.111/6.147, 808 Route de Lennik, B-1070 Brussels, Belgium. E-mail: pvdhaegh@ulb.ac.be.

DOI:10.1523/JNEUROSCI.0802-05.2005

Copyright $\odot 2005$ Society for Neuroscience $\quad$ 0270-6474/05/257232-06\$15.00/0 tectum or SC are expressed in complementary gradients. Neurons from the temporal retina project to the anterior tectum or $\mathrm{SC}$, whereas axons from the nasal retina project to the posterior tectum, mainly through repulsive guidance effects of ephrin-As on EphA-expressing axons, as well as axon-axon competition (Frisen et al., 1998; Brown et al., 2000; Feldheim et al., 2000, 2004). Work in the mouse has shown that similar mechanisms operate to map retinal axons onto the dorsal lateral geniculate nucleus (dLGN) in the thalamus, the main retinal target in most mammals (Feldheim et al., 1998) (see Fig. 1A).

In most model organisms, all axons from each retina project to the contralateral part of the brain, except in Xenopus frog and mouse, in which a small subset of ventral retinal axons project to the ipsilateral brain (Jeffery, 2001; Williams et al., 2004). This ipsilateral routing is mediated in part by $\mathrm{EphB}$ receptors, which are selectively expressed on axons from this subquadrant and render them sensitive to repulsive effects of ephrin-B2 expressed in the optic chiasm (Nakagawa et al., 2000; Williams et al., 2003; Klein, 2004) (see Fig. 1A).

However, several mammals, in particular primates, display an entirely binocular organization of their retinal projections, in which all axons from each nasal hemi-retina project to the contralateral dLGN, whereas all axons from each temporal hemiretina project ipsilaterally (Guillery, 1996; Jeffery, 2001). Axons from each hemi-retina segregate from each other within defined domains corresponding to dLGN eye-specific layers, and each hemi-retina is connected topographically to its corresponding layers, thereby generating congruent retinotopic maps that are aligned in alternating layers (see Fig. $1 B$ ). 
This anatomical divergence has important functional consequences, because it constitutes the primary basis for binocular stereoscopic vision. It also raises several developmental and evolutionary issues on the roles of mapping labels including ephrin/ Eph genes in the emergence of binocular maps. Indeed, if ephrin/ Eph genes were to be involved in retinotopic mapping in binocular species, they should be part of a distinct system of labels to account for the congruent mapping of temporal and nasal parts of each retina onto the dLGN (see Fig. 1). Alternatively, if they retained the same nasal-temporal distribution in binocular species, they could control other aspects of retinal projections, such as the segregation of ipsilateral (temporal) and contralateral (nasal) retinal fibers within distinct layers of the dLGN, which is likely to depend on (yet uncharacterized) mapping labels as well as on patterned neural activity (Katz and Shatz, 1996; Crowley and Katz, 2000; Crair et al., 2001; Katz and Crowley, 2002; Cline, 2003; Huberman et al., 2003).

In addition to binocularity, the patterning of retinal projections displays other important divergent features in primates. In particular, the development of primate retinal topography and eye segregation, unlike in the cat or ferret for instance, seems to be characterized by an early emergence of fiber ordering, with little exuberance and pruning (Chalupa et al., 1996; Meissirel et al., 1997; Snider et al., 1999; Wefers et al., 2000), raising further the question of the evolutionary conservation of the mechanisms of development of retinal projections.

To try to address these issues, we looked at the expression patterns of ephrin/Eph genes in the human developing retinogeniculate system and found that their distribution is fundamentally divergent from what was previously described in model organisms.

\section{Materials and Methods}

Cases studied. Expression patterns of ephrin/Eph genes in the eye were studied in nine human fetuses obtained after voluntary pregnancy termination and evenly distributed between 40 and 56 postovulatory days and in six human fetuses of $10,10.5,11,19,21$, and 24 weeks gestational age (GW). The expression profile in the LGN was studied in four fetuses of $11,14,17$, and $19 \mathrm{GW}$. Fetuses with conditions leading to potential eye or brain anomalies were excluded from the study. The study was approved by the three relevant local Ethics Committees (Erasme Hospital, University of Brussels, and Belgian National Fund for Scientific Research) on research involving human subjects. Written informed consent was given by the parents in each case.

In situ RNA hybridization. Human probes for ephrin-A5 (corresponding to nucleotides 332-1166; GenBank accession number NM001962), EphA5 (nucleotides 850-1692; accession number NM004439), EphA6 (nucleotides 945-1560; accession number XM114973), EphA7 (nucleotides 1488-2083; accession number NM004440), ephrin-B2 (nucleotides 460-1208; accession number NM004093), and EphB1 (nucleotides 1327-2106; accession number NM004441) were obtained by subcloning reverse transcription-PCR products amplified from human fetal brain cDNA (primer sequences available on request). Heads or dissected brains and eyes were frozen freshly in cooled $\left(-70^{\circ} \mathrm{C}\right)$ isopentane. Cryosections of $20 \mu \mathrm{m}$ were obtained using a customized Leica (Nussloch, Germany) CM3000 cryostat. In situ hybridization using digoxigenin-labeled RNA probes was performed as described previously (Vanderhaeghen et al., 2000 ). Four to 20 sections from each case were hybridized to each probe described.

For semiquantitative analysis of ephrin/Eph gene expression patterns in the retina, we used the following procedure: for each eye section, a digitized image was taken (with the same exposition parameters), and for each of these, the retina was divided into 19 equal quadrants. The mean pixel intensity was then measured (using Photoshop) in each quadrant at the level of the retinal ganglion cell layer. This measurement was done on four different eyes (two to five sections for each eye and for each probe) at $10 \mathrm{GW}$ (two eyes) and 10.5 GW (two eyes).
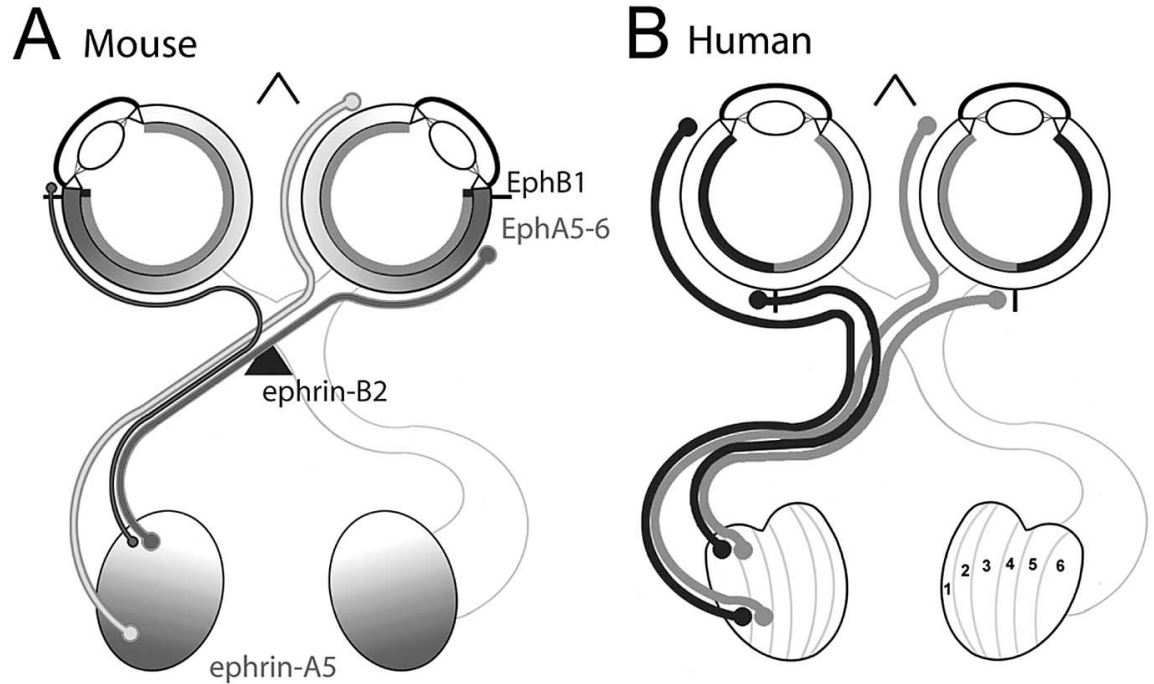

Figure 1. Schematic model comparing visual mapping in the mouse and human. $\boldsymbol{A}$, In the mouse, axons from each retina project to the contralateral part of the brain, except a small contingent of axons from the ventrotemporal retina that project ipsilaterally. EphA receptors (gray) are expressed in a high temporal to low nasal gradient in the retina, whereas EphB1 is restricted to a small ventrotemporal crescent (black). Most axons cross the midline [with the exception of the small contingent of EphB1expressing axons that are repelled by ephrin-B2 in the optic chiasm (black triangle)] and map onto the dLGN where ephrin-As are displayed in a single complementary gradient. $\boldsymbol{B}$, In the human, retinal projections display a fully binocular organization, such that all axons from each nasal hemi-retina (light gray) project to the contralateral dLGN, whereas all axons from each temporal hemi-retina (black) project ipsilaterally. Axons from each hemi-retina segregate from each other within defined domains corresponding to dLGN eye-specific layers (1-6), and each hemi-retina is connected topographically to its corresponding layers, thereby generating congruent retinotopic maps that are aligned in alternating layers.

\section{Results}

We focused on ephrin/Eph genes known to be involved in retinal axon guidance and mapping in model organisms (ephrin-A5, ephrin-B2, EphA5, EphA6, EphA7, and EphB1), at relevant stages in the human developing primary visual system, corresponding to the invasion of the dLGN by retinal fibers $(7 \mathrm{GW})$ until segregation of ipsilateral and contralateral retinal fibers (20 GW) (Hevner, 2000).

When looking at the pattern of expression of ephrin/Eph genes in the human developing retina, we found that their distribution was fundamentally different from what has been described in all model organisms, at all stages examined (see Fig. 2). Both EphA5 and EphA6 were found to be expressed in a bidirectional gradient, with its peak located temporally close to the central retina (corresponding to the presumptive fovea), sloping down toward the nasal and temporal poles of the peripheral retina (see Fig. 2A-E). Ephrin-A5 was expressed in a complementary manner, also along two gradients, high peripheral to low central (see Fig. 2B-E). A similar central to peripheral pattern of expression was observed throughout all stages examined, from 8-9 GW to 19-21 GW (see Fig. 
$2 B-E$ and data not shown). No expression in the retina could be detected for EphA7 (data not shown).

Interestingly, the temporal and nasal arms of the gradients appeared to be slightly asymmetrical, with EphA receptors expressed at a higher level in the temporal retina and ephrin-A5 expressed at a higher level in the nasal retina (see Fig. $2 B-D$ ).

EphB1 was found to be expressed differently, at a high level throughout the temporal retina, its level falling abruptly on the nasal side of the retina (see Fig. $2 C, D)$. Interestingly, the site of shift from high to low EphB1 expression corresponded to the location of the peak of expression of EphA receptors around the center of the retina (see Fig. $2 C, D$ ). Because the pattern of expression of EphB1 matches the divergent pattern of projections found in primates, in which the entire temporal retina projects ipsilaterally (Fig. 1B), we also checked for the expression of ephrin-B2, which was found in the developing optic chiasm (Fig. $2 F$ ), as described previously in other vertebrates (Fig. 1A) (Nakagawa et al., 2000; Williams et al., 2003, 2004).

We next turned to the pattern of expression of the same genes in the dLGN. At each stage examined, ephrin-A5 was expressed in a high lateroventral to low mediodorsal gradient, whereas EphA7 was expressed in a mirror manner, higher dorsally and lower ventrally (Fig. 3 ). This distribution along a single gradient is similar to the one described previously in the mouse dLGN, which follows the lines of retinotopy (from temporal to nasal) (Feldheim et al., 1998). The complementarity of the ephrin-A5 and EphA7 patterns of expression is also strikingly reminiscent of the patterns found in the mouse brain (Yun et al., 2003; Depaepe et al., 2005). Importantly the ephrin-A5/EphA7 gradients also follow the main axis of retinotopy (from center to periphery) described in the developing human and macaque (Rakic, 1977; Hitchcock and Hickey, 1980; Meissirel et al., 1997; Snider et al., 1999; Hevner, 2000). Similarly, when comparing different anteroposterior levels of the dLGN, ephrin-A5 was found to be expressed at the highest levels anteriorly, whereas EphA7 was expressed at the highest levels posteriorly (data not shown).

Again, this topographic arrangement corresponds to the axes of retinotopy (from center to periphery) described in the primate dLGN (Connolly and Van Essen, 1984; Schneider et al., 2004).

\section{Discussion}

Here, we show that the distribution of ephrin-A/EphA genes in the human developing retina is fundamentally different from
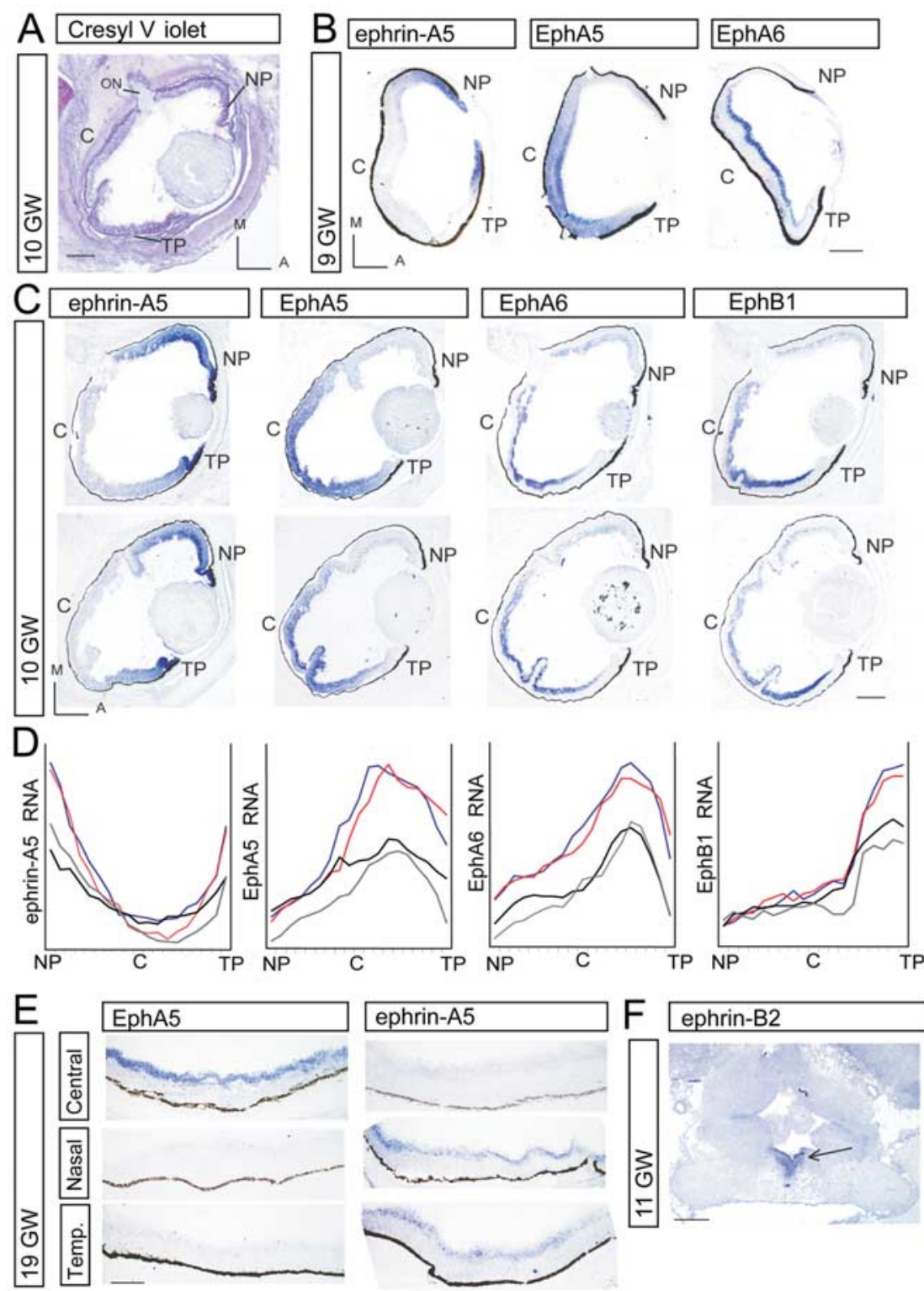

Figure 2. Pattern of expression of ephrin/Eph genes in the human developing retina. $A$, Cresyl violet-stained horizontal section of a $10 \mathrm{GW}$ human fetal retina illustrating the basic topology of the eye, with the optic nerve (ON), the center of the retina, the nasal peripheral retina, and the temporal peripheral retina. $\boldsymbol{B}, \boldsymbol{C}$, In situ hybridization on $9 \mathrm{GW}(\boldsymbol{B})$ and $10 \mathrm{GW}(\boldsymbol{C})$ human eye horizontal sections (anterior is to the right, and medial is at the top). EphA5 and EphA6 are expressed along two gradients, high central to low peripheral. Ephrin-A5 is expressed in a complementary manner along two gradients, high peripheral to low central. EphB1 is expressed at the highest levels in a temporal crescent decreasing at around the same location as the central peak of expression of EphA5-6. D, Densitometric scans illustrating the expression pattern of the four genes in the retina. Each panel shows densitometry of horizontal sections of four human eyes (for each probe, each colored curve represents the means of densitometric measurements performed on $2-5$ sections for each eye). The $x$-axis represents 19 quadrants of the retina from its nasal peripheral edge to its temporal (Temp.) peripheral edge, and the $y$-axis represents relative RNA levels in arbitrary units. $\boldsymbol{E}$, In situ hybridization on a human eye horizontal section at $19 \mathrm{GW}$, illustrating the maintenance of the center to periphery gradients of ephrin-A5 and EphA5/EphA6 at this later stage. $\boldsymbol{F}$, In situ hybridization on an $11 \mathrm{GW}$ human brain coronal section, illustrating selective expression of ephrin-B2 at the level of the optic chiasm (arrow). Scale bars: $A, C, F, 500 \mu \mathrm{m} ; \boldsymbol{B}, \boldsymbol{E}, 200 \mu \mathrm{m}$. C, Center of the retina; NP, nasal peripheral retina; $T P$, temporal peripheral retina; $A$, anterior; $M$, medial.

what has been described in all model organisms examined so far (Fig. $4 \mathrm{~A}$ ), because they are displayed along two gradients, which follow a center to periphery retinotopic arrangement. In contrast, the distribution of ephrin/Eph genes in the dLGN looks quite similar to the one described in the mouse (Feldheim et al., 1998) and follows the lines of projection of retinotopy, from center to periphery, described in developing and adult primates (Rakic, 


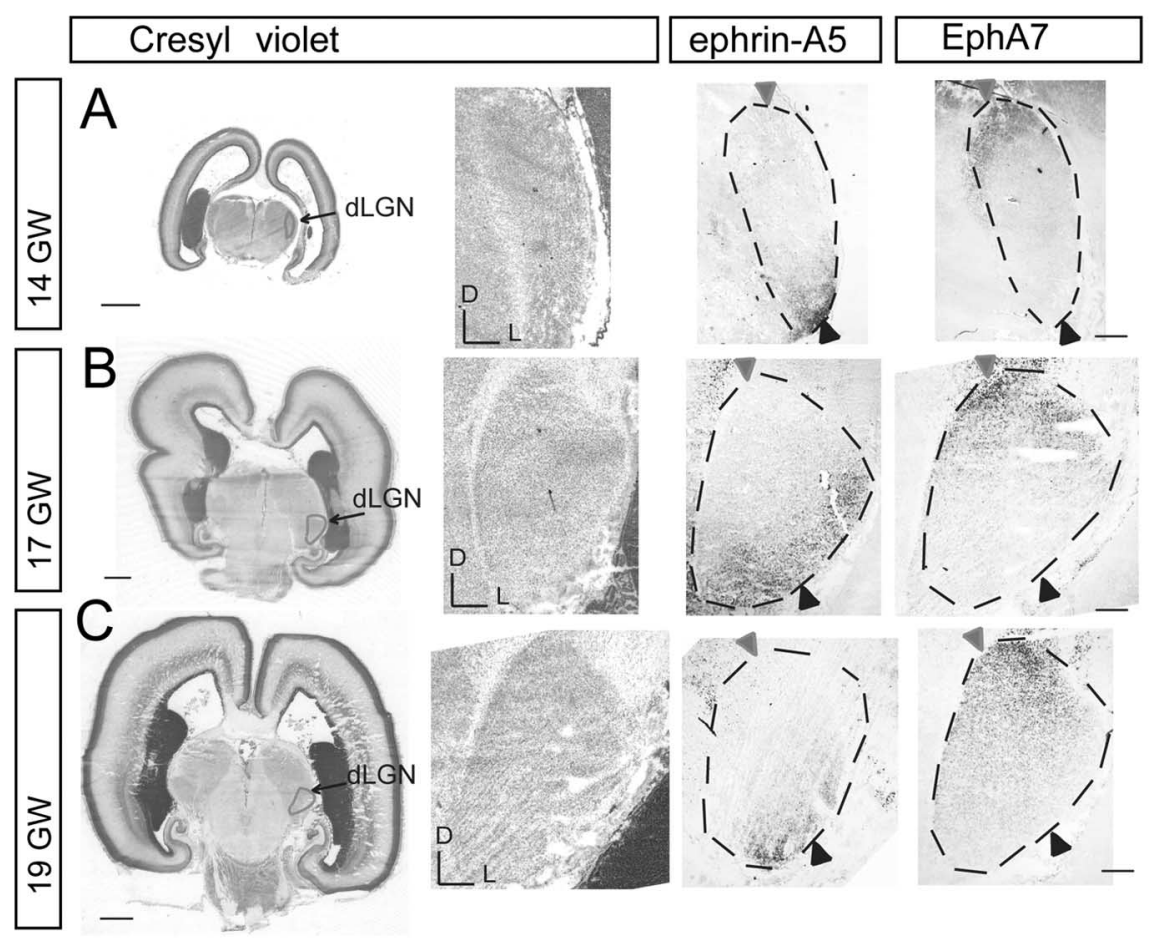

Figure 3. Patterns of expression of ephrin/Eph genes in the human developing dLGN. $\boldsymbol{A}-\boldsymbol{C}$, Coronal sections of $14 \mathrm{GW}(\boldsymbol{A}), 17$ $\mathrm{GW}(\boldsymbol{B})$, and $19 \mathrm{GW}(\boldsymbol{C})$ human fetal dorsal lateral geniculate nuclei (the location of the nucleus is delineated on low-magnification views in the left panels and with black dashed lines on in situ hybridization panels) stained for cresyl violet (left) or hybridized to the indicated probes (right). At each stage, ephrin-A5 is expressed in a high ventrolateral (black arrowhead) to low mediodorsal (gray arrowhead) pattern, whereas EphA7 is expressed in a mirror manner, highest dorsomedially and lowest ventrolaterally. Dorsal (D) is at the top, and lateral $(\mathrm{L})$ is to the right. Scale bars: $500 \mu \mathrm{m}$; whole sections (extreme left), $5 \mathrm{~mm}$.
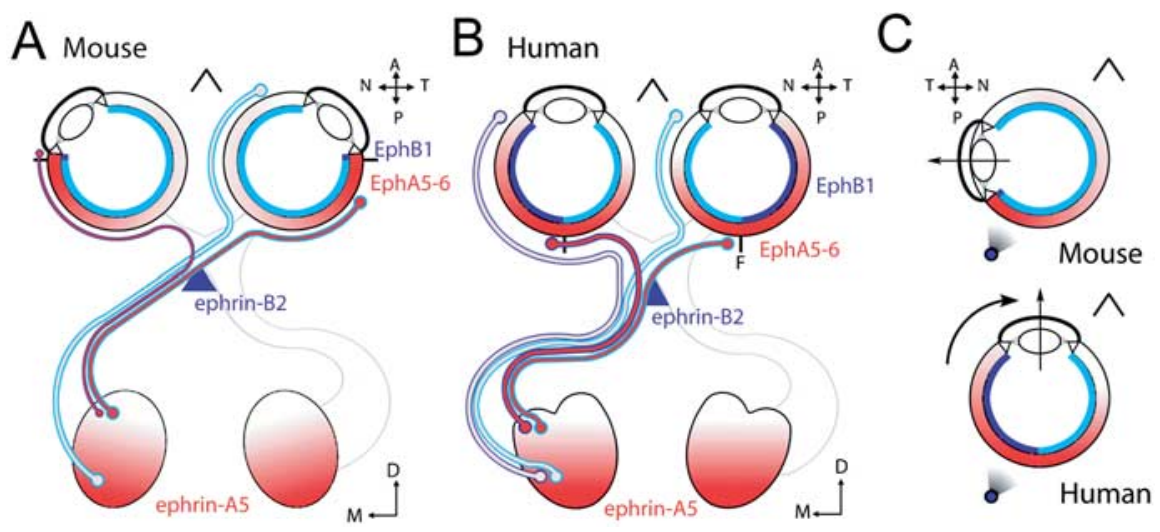

Figure 4. Schematic model comparing visual mapping and ephrin/Eph gene distribution in the mouse and human. $\boldsymbol{A}$, In the mouse retina, EphA receptors (red) are expressed in a high temporal to low nasal gradient, whereas EphB1 is restricted to a small ventrotemporal crescent (dark blue). Most axons cross the midline [with the exception of the small contingent of EphB1expressing axons that are repelled by ephrin-B2 in the optic chiasm (blue triangle)] and map onto the dLGN where ephrin-As are displayed in a single complementary gradient. $\boldsymbol{B}$, In the human retina, EphA receptors (red) are displayed in a bidirectional gradient, from the presumptive foveal center $(F)$ to the nasal and temporal periphery, whereas EphB1 is displayed at a high level of expression throughout the temporal retina, which abruptly decreases from the foveal center to the nasal retina. Each temporal and nasal hemi-retina reaches its contralateral and ipsilateral targets, in part through EphB1/ephrin-B2 interactions at the optic chiasm. Within the dLGN, a single complementary gradient of ephrin-As controls the congruent mapping of retinal axons from both eyes, which will become segregated into distinct layers (not yet visible at this stage of development) through as yet unidentified mechanisms. $\boldsymbol{C}$, Tentative evolutionary model explaining the coordination of changes in the patterning of the retina after eye rotation in binocular species. A morphogenic center (dark point) located outside of the retina would signal to control the expression of Eph genes in the temporal pole of the mouse retina. During the emergence of binocularity, the rotation of the eye would change the spatial relationships between this "fixed" center and the retina, because it would now lie closer to the retinal center, thereby changing accordingly the patterning of ephrin/Eph gene expression. Dorsal (D) is at the top, and medial (M) is to the left. N, Nasal; A, anterior; T, temporal; P, posterior.
1977; Hitchcock and Hickey, 1980; Connolly and Van Essen, 1984; Meissirel et al., 1997; Snider et al., 1999; Hevner, 2000; Schneider et al., 2004).

This distribution of ephrin/Eph genes in the retina and dLGN, characterized by complementary, center to periphery, gradients is ideally suited to control the congruent mapping of axons from each hemiretina onto the dLGN (Fig. $1 B$ ), thus strongly suggesting their implication in binocular retinotopic mapping, as schematized in Figure 4B. Remarkably, a similar center to periphery arrangement of mapping labels in primates was already hypothesized by Sperry (1963) to account for the generation of congruent binocular maps.

On the other hand, our data seem to argue against a role for ephrin-As in the segregation of retinal axons within eyespecific layers, because ephrin-A/EphA gradients in the dLGN essentially follow the lines of projection of retinotopy, and are thus parallel to the orientation of presumptive eye-specific layers (Rakic, 1977; Hitchcock and Hickey, 1980; Hevner, 2000). However, the asymmetrical pattern of expression of ephrin-A and EphA genes observed within the nasal and temporal peripheral retinas (Fig. 2B,C) could provide a substrate for axon-axon segregation within the optic tract (Meissirel and Chalupa, 1994) or in the dLGN, thereby contributing to the formation or maintenance of eye-specific layers. Indeed, axon-axon interactions were proposed previously to participate in retinotopic mapping in monocular species, in which ephrin-As and EphAs are also expressed in countergradients within the retina (Hornberger et al., 1999; Knoll and Drescher, 2002). It also remains conceivable that ephrin/Eph genes display a different pattern of expression later in development that could account for additional roles in the development or plasticity of layering of retinal projections. Notably, such a dual role for ephrin/Eph genes in controlling sequential steps of generation of connectivity was already shown in the mouse thalamocortical system (Dufour et al., 2003; Vanderhaeghen and Polleux, 2004).

Another divergent feature of the human system is that EphB1 is expressed throughout the temporal retina, in correlation with the fact that the entire temporal retina remains ipsilateral in the human species (Fig. 4B). Together with our observation that ephrin-B2 is expressed at the site of the developing optic chiasm, this strongly suggests the crucial role of ephrin$B / E p h B$ genes in the differential control of 
ipsilateral routing of axons at the optic chiasm in the human, as shown previously in model organisms (Williams et al., 2004). The fact that EphB1 is expressed at a higher level throughout the temporal half of the retina in the human raises the question of its potential influence on the mechanisms controlling dorsoventral mapping, which is thought to be controlled by ephrin-B/EphB in the mouse and Xenopus (McLaughlin et al., 2003). This issue was already brought up by studies in the mouse, in which EphB1 is selectively expressed in axons from the ventrotemporal retina, whereas ephrin-B1-2 and EphB2-3 are expressed along complementary dorsoventral gradients in the retina and tectum. Several mechanisms have been proposed to explain this apparent discrepancy, including differential posttranscriptional control or modulation of EphB signaling by ephrin-B coexpression (Williams et al., 2003). In this context, it would be very interesting to look in more detail at the distribution of ephrin-B/EphB genes in the human, both at the RNA and protein levels, to test whether dorsoventral mapping essentially follows the same rules as in less binocular species, despite the divergence in expression of EphB1.

Strikingly, the peak of EphA expression in the center of the retina matches the abrupt site of shift of expression of EphB1 (Fig. $2 C, D)$. Such a coordinated change in the patterns of expression of the genes controlling retinal axon crossing at the optic chiasm and retinotopic mapping is likely to have provided the developmental basis for the emergence of binocularity during evolution (Fig. 4A,B). The coordinated evolution of the patterns of expression of ephrin/Eph genes observed in a binocular species raises the question of the upstream mechanisms involved. A parsimonious model would be that an organizing/morphogenetic center located outside of the retina but close to its temporal pole would be responsible for the control of EphA gradients and EphB1 expression in the temporal retina of monocular species (Fig. 4C). During the emergence of binocularity, the orientation of the developing eye would rotate (with its main axis, from the center of the retina to the center of the lens, becoming more parallel to the midline) with respect to this fixed extra-retinal center, so that it would lie closer to the presumptive fovea of the retina and further from the temporal pole (Fig. $4 C$ ). Thus, the rotation of the eye (which per se constitutes the primary basis for binocular vision) would be tightly coordinated with the changes of pattern of expression of EphB1 and EphA receptors necessary for the generation of a binocular system (Fig. 4C). In this context, it will be most interesting to look in the human, and other species displaying various degrees of binocularity, at the patterns of expression of the transcription factors that were recently found to control retinal projections and expression of ephrin/Eph genes in the mouse and chick retina (Herrera et al., 2003; McLaughlin et al., 2003; Pak et al., 2004; Williams et al., 2004).

Finally, this study constitutes the first demonstration that ephrin/Eph axon guidance cues, previously known to be important in the generation of neuronal connectivity in model organisms, are also involved in the development of human brain networks. This may provide physiopathological insight on neurological conditions characterized by impaired development of sensory function: for instance, any developmental alteration of the tight coordination between axon guidance at the chiasm and retinal mapping, including disruption of ephrin/Eph pathways, may result in impairment of visual processing (Williams et al., 1994; Apkarian et al., 1995; Guillery, 1996).

\section{References}

Apkarian P, Bour LJ, Barth PG, Wenniger-Prick L, Verbeeten Jr B (1995) Non-decussating retinal-fugal fibre syndrome. An inborn achiasmatic malformation associated with visuotopic misrouting, visual evoked potential ipsilateral asymmetry and nystagmus. Brain 118:1195-1216.

Brown A, Yates PA, Burrola P, Ortuno D, Vaidya A, Jessell TM, Pfaff SL, O'Leary DD, Lemke G (2000) Topographic mapping from the retina to the midbrain is controlled by relative but not absolute levels of EphA receptor signaling. Cell 102:77-88.

Chalupa LM, Meissirel C, Lia B (1996) Specificity of retinal ganglion cell projections in the embryonic rhesus monkey. Perspect Dev Neurobiol 3:223-231.

Cline H (2003) Sperry and Hebb: oil and vinegar? Trends Neurosci 26:655-661.

Connolly M, Van Essen D (1984) The representation of the visual field in parvicellular and magnocellular layers of the lateral geniculate nucleus in the macaque monkey. J Comp Neurol 226:544-564

Crair MC, Horton JC, Antonini A, Stryker MP (2001) Emergence of ocular dominance columns in cat visual cortex by 2 weeks of age. J Comp Neurol 430:235-249.

Crowley JC, Katz LC (2000) Early development of ocular dominance columns. Science 290:1321-1324.

Depaepe V, Suarez-Gonzalez N, Dufour A, Passante L, Gorski JA, Jones KR, Ledent C, Vanderhaeghen P (2005) Ephrin signalling controls brain size by regulating apoptosis of neural progenitors. Nature 435:1244-1250.

Drescher U (2004) Aber Lenken Sie Auch? (But do they also guide?) J Neurobiol 59:3-7.

Dufour A, Seibt J, Passante L, Depaepe V, Ciossek T, Frisen J, Kullander K, Flanagan JG, Polleux F, Vanderhaeghen P (2003) Area specificity and topography of thalamocortical projections are controlled by ephrin/Eph genes. Neuron 39:453-465.

Feldheim DA, Vanderhaeghen P, Hansen MJ, Frisen J, Lu Q, Barbacid M, Flanagan JG (1998) Topographic guidance labels in a sensory projection to the forebrain. Neuron 21:1303-1313.

Feldheim DA, Kim YI, Bergemann AD, Frisen J, Barbacid M, Flanagan JG (2000) Genetic analysis of ephrin-A2 and ephrin-A5 shows their requirement in multiple aspects of retinocollicular mapping. Neuron 25:563-574.

Feldheim DA, Nakamoto M, Osterfield M, Gale NW, DeChiara TM, Rohatgi R, Yancopoulos GD, Flanagan JG (2004) Loss-of-function analysis of EphA receptors in retinotectal mapping. J Neurosci 24:2542-2550.

Flanagan JG, Vanderhaeghen P (1998) The ephrins and Eph receptors in neural development. Annu Rev Neurosci 21:309-345.

Frisen J, Yates PA, McLaughlin T, Friedman GC, O’Leary DD, Barbacid M (1998) Ephrin-A5 (AL-1/RAGS) is essential for proper retinal axon guidance and topographic mapping in the mammalian visual system. Neuron 20:235-243.

Guillery RW (1996) Why do albinos and other hypopigmented mutants lack normal binocular vision, and what else is abnormal in their central visual pathways? Eye 10: 217-221.

Herrera E, Brown L, Aruga J, Rachel RA, Dolen G, Mikoshiba K, Brown S, Mason CA (2003) Zic2 patterns binocular vision by specifying the uncrossed retinal projection. Cell 114:545-557.

Hevner RF (2000) Development of connections in the human visual system during fetal mid-gestation: a DiI-tracing study. J Neuropathol Exp Neurol 59:385-392.

Hitchcock PF, Hickey TL (1980) Prenatal development of the human lateral geniculate nucleus. J Comp Neurol 194:395-411.

Hornberger MR, Dutting D, Ciossek T, Yamada T, Handwerker C, Lang S, Weth F, Huf J, Wessel R, Logan C, Tanaka H, Drescher U (1999) Modulation of EphA receptor function by coexpressed ephrinA ligands on retinal ganglion cell axons. Neuron 22:731-742.

Huberman AD, Wang GY, Liets LC, Collins OA, Chapman B, Chalupa LM (2003) Eye-specific retinogeniculate segregation independent of normal neuronal activity. Science 300:994-998.

Jeffery G (2001) Architecture of the optic chiasm and the mechanisms that sculpt its development. Physiol Rev 81:1393-1414.

Katz LC, Crowley JC (2002) Development of cortical circuits: lessons from ocular dominance columns. Nat Rev Neurosci 3:34-42.

Katz LC, Shatz CJ (1996) Synaptic activity and the construction of cortical circuits. Science 274:1133-1138.

Klein R (2004) Eph/ephrin signaling in morphogenesis, neural development and plasticity. Curr Opin Cell Biol 16:580-589.

Knoll B, Drescher U (2002) Ephrin-As as receptors in topographic projections. Trends Neurosci 25:145-149. 
McLaughlin T, Hindges R, O'Leary DD (2003) Regulation of axial patterning of the retina and its topographic mapping in the brain. Curr Opin Neurobiol 13:57-69.

Meissirel C, Chalupa LM (1994) Organization of pioneer retinal axons within the optic tract of the rhesus monkey. Proc Natl Acad Sci USA 91:3906-3910.

Meissirel C, Wikler KC, Chalupa LM, Rakic P (1997) Early divergence of magnocellular and parvocellular functional subsystems in the embryonic primate visual system. Proc Natl Acad Sci USA 94:5900-5905.

Nakagawa S, Brennan C, Johnson KG, Shewan D, Harris WA, Holt CE (2000) Ephrin-B regulates the ipsilateral routing of retinal axons at the optic chiasm. Neuron 25:599-610.

Pak W, Hindges R, Lim YS, Pfaff SL, O’Leary DD (2004) Magnitude of binocular vision controlled by islet- 2 repression of a genetic program that specifies laterality of retinal axon pathfinding. Cell 119:567-578.

Rakic P (1977) Genesis of the dorsal lateral geniculate nucleus in the rhesus monkey: site and time of origin, kinetics of proliferation, routes of migration and pattern of distribution of neurons. J Comp Neurol 176:23-52.

Schneider KA, Richter MC, Kastner S (2004) Retinotopic organization and functional subdivisions of the human lateral geniculate nucleus: a highresolution functional magnetic resonance imaging study. J Neurosci 24:8975-8985.

Snider CJ, Dehay C, Berland M, Kennedy H, Chalupa LM (1999) Prenatal development of retinogeniculate axons in the macaque monkey during segregation of binocular inputs. J Neurosci 19:220-228.

Sperry R (1963) Chemoaffinity in the orderly growth of nerve fiber patterns and connections. Proc Natl Acad Sci USA 50:703-710.

Vanderhaeghen P, Polleux F (2004) Developmental mechanisms patterning thalamocortical projections: intrinsic, extrinsic and in between. Trends Neurosci 27:384-391.

Vanderhaeghen P, Lu Q, Prakash N, Frisen J, Walsh CA, Frostig RD, Flanagan JG (2000) A mapping label required for normal scale of body representation in the cortex. Nat Neurosci 3:358-365.

Wefers CJ, Dehay C, Berland M, Kennedy H, Chalupa LM (2000) Binocular competition does not regulate retinogeniculate arbor size in fetal monkey. J Comp Neurol 427:362-369.

Williams RW, Hogan D, Garraghty PE (1994) Target recognition and visual maps in the thalamus of achiasmatic dogs. Nature 367:637-639.

Williams SE, Mann F, Erskine L, Sakurai T, Wei S, Rossi DJ, Gale NW, Holt CE, Mason CA, Henkemeyer M (2003) Ephrin-B2 and EphB1 mediate retinal axon divergence at the optic chiasm. Neuron 39:919-935.

Williams SE, Mason CA, Herrera E (2004) The optic chiasm as a midline choice point. Curr Opin Neurobiol 14:51-60.

Yun ME, Johnson RR, Antic A, Donoghue MJ (2003) EphA family gene expression in the developing mouse neocortex: regional patterns reveal intrinsic programs and extrinsic influence. J Comp Neurol 456: 203-216. 